\title{
Prevalence, risk factors and multilocus genotyping of Enterocytozoon bieneusi in farmed foxes (Vulpes lagopus), Northern China
}

\author{
Xiao-Xuan Zhang ${ }^{1,2+}$, Wei Cong ${ }^{1,2+}$, Zhi-Long Lou ${ }^{1,2}$, Jian-Gang Ma ${ }^{1,2}$, Wen-Bin Zheng ${ }^{1,2}$, Qiu-Xia Yao ${ }^{1,3}$, \\ Quan Zhao ${ }^{2}$ and Xing-Quan Zhu ${ }^{1,2,3^{*}}$
}

\begin{abstract}
Background: Microsporidiosis is a common disease in animals and humans around the world. Enterocytozoon bieneusi is the most common microsporidian species in humans. Many animal species may be a potential source of human microsporidiosis. However, information concerning prevalence and genotypes of E. bieneusi infection in farmed foxes (Vulpes lagopus) is scarce. Therefore, the present study examined prevalence, risk factors and genotypes of $E$. bieneusi in farmed foxes in northern China using a genetic approach.

Results: Of 302 fecal samples from farmed foxes, 37 (12.25\%, $95 \%$ Cl 8.55-15.95) were PCR-positive for E. bieneusi, and the prevalence was highly associated with the farming mode in that foxes raised outdoors $(26.03 \%$ positive, $95 \% \mathrm{Cl} 18.91-33.15)$ had a significantly higher E. bieneusi prevalence than those raised indoors. Eleven internal transcribed spacer (ITS) genotypes were identified among the positive samples: four known E. bieneusi genotypes (Peru 8, Types IV, CHN-DC1 and D) and seven novel genotypes (NCF1-NCF7). Genotype NCF2 was the commonest $(n=13)$ and was found in five farms across three provinces (Jilin, Heilongjiang and Hebei). All genotypes belonged to phylogenetic group 1. Multilocus sequence typing (MLST) analyses revealed additional diversity.

Conclusions: These findings indicate the presence of zoonotic E. bieneusi infection in farmed foxes in northern China. This is also the first report of genotypes Peru8, CHN-DC1 and Type IV, and seven novel genotypes (NCF1-NCF7) in farmed foxes by ITS combining with microsatellite and minisatellite markers for the first time. The results will provide baseline data for preventing and controlling E. bieneusi infection in farmed foxes, other animals and humans.
\end{abstract}

Keywords: Enterocytozoon bieneusi, Foxes (Vulpes lagopus), Prevalence, Genotyping, Northern China

\section{Background}

Microsporidia form an important group of obligate intracellular parasites, and the 1300 named species can infect virtually all animals [1-4]. Fourteen species in eight genera have been reported in humans [5]. Enterocytozoon bieneusi, Encephalitozoon cuniculi, Encephalitozoon intestinalis, and

\footnotetext{
* Correspondence: xingquanzhu1@hotmail.com

${ }^{\dagger}$ Equal contributors

'State Key Laboratory of Veterinary Etiological Biology, Key Laboratory of Veterinary Parasitology of Gansu Province, Lanzhou Veterinary Research Institute, Chinese Academy of Agricultural Sciences, Lanzhou, Gansu Province 730046, PR China

${ }^{2}$ College of Animal Science and Technology, Jilin Agricultural University, Changchun, Jilin Province 130118, PR China

Full list of author information is available at the end of the article
}

Encephalitozoon hellem are the major microsporidians infecting humans worldwide [5], with $E$. bieneusi being the most common, responsible for $90 \%$ of all microsporidian infections [6]. The first case of human infection with $E$. bieneusi was reported in an AIDS patient with chronic diarrhea [6, 7]. Since E. bieneusi was detected in pig feces, animals have slowly become recognized as the main intermediate hosts of this pathogen $[4,8]$. Feces containing E. bieneusi spores can contaminate water or food, leading to microsporidiosis outbreaks [9].

The internal transcribed spacer (ITS) region of the ribosomal RNA (rRNA) gene cluster has been widely 
used to evaluate the zoonotic risk of E. bieneusi [10-13]. Thus far, two large groups have been documented by phylogenetic analysis of ITS sequences, namely a zoonotic group (Group 1) which can infect both humans and animals, and a number of host-adapted groups (Groups 2-5 and outlier genotypes in dogs). More than 200 distinct non-human genotypes are known [14-18]. Some additional small groups have also been found in recent years: Group 7 in Nigerian AIDS patients, Group 6 in urban wastewater in China, and Group 8 was also found in some animals in recent studies $[19,20]$.

China has abundant animal resources, but only limited information is available regarding prevalence and genotypes of E. bieneusi. Most studies in China have reported on E. bieneusi in pigs, dogs, cats, cattle, sheep, humans, captive snakes, pandas, some nonhuman primates, and environmental samples [13, 14, 17, 21-26]. Foxes have been the subject of only one study, which was conducted in Harbin City [27]. To improve the information of the distribution of $E$. bieneusi genotypes and estimate the zoonotic potential of E. bieneusi infection in foxes, a cross-sectional study of 302 farmed foxes (Vulpes lagopus) in northern China was conducted.

\section{Methods}

\section{Specimen collection and DNA extraction Ethical statement}

This study was approved by the Animal Ethics Committee of Lanzhou Veterinary Research Institute, Chinese Academy of Agricultural Sciences (Approval No. LVR IAEC2014-011). In total, 302 fecal samples were randomly collected from healthy farmed foxes from Jilin province (3 farms, 91 foxes), Heilongjiang province ( 2 farms, 70 foxes) and Hebei province (3 farms, 141 foxes), northern China in 2014. Each fecal sample was collected using sterile gloves immediately after the animal had defecated, and was then stored on ice. In the laboratory, genomic DNA was immediately extracted from feces using an EZNAR Stool DNA kit (OMEGA, USA) according to the manufacturer's instructions and stored at $-20{ }^{\circ} \mathrm{C}$ until required for PCR analyses. Information regarding geographical origin, farming mode, gender and age of source fox were acquired for each fecal sample.

\section{PCR amplification}

The genotypes/subtypes of E. bieneusi in farmed foxes in northern China were determined using nested PCRs with primers listed in Table 1 [13]. Each reaction $(25 \mu \mathrm{l})$ contained 1 x Ex Taq buffer $\left(\mathrm{Mg}^{2+}\right.$ free), $2 \mathrm{mM} \mathrm{MgCl}_{2}$,

Table 1 Primers used in the study, annealing temperatures used in the PCRs and expected sizes of the PCR products

\begin{tabular}{|c|c|c|c|c|c|}
\hline Gene & Primer & Sequence $(5-3)$ & Annealing temperature $\left({ }^{\circ} \mathrm{C}\right)$ & Fragment length (bp) & References \\
\hline \multirow[t]{4}{*}{ ITS } & F1 & GGTCATAGGGATGAAGAG & 55 & 392 & 13 \\
\hline & R1 & TTCGAGTTCTITCGCGCTC & & & \\
\hline & $\mathrm{F} 2$ & GCTCTGAATATCTATGGCT & 55 & & \\
\hline & R2 & ATCGCCGACGGATCCAAGTG & & & \\
\hline \multirow[t]{4}{*}{ MS1 } & F1 & CAAGTTGCAAGTTCAGTGTTTGAA & 58 & 675 & 13 \\
\hline & R1 & GATGAATATGCATCCATTGATGTT & & & \\
\hline & F2 & TTGTAAATCGACCAAATGTGCTAT & 58 & & \\
\hline & R2 & GGACATAAACCACTAATTAATGTAAC & & & \\
\hline \multirow[t]{4}{*}{ MS3 } & F1 & CAAGCACTGTGGTTACTGTT & 55 & 537 & 13 \\
\hline & R1 & AAGTTAGGGCATTTAATAAAATTA & & & \\
\hline & F2 & GTTCAAGTAATTGATACCAGTCT & 55 & & \\
\hline & R2 & CTCATTGAATCTAAATGTGTATAA & & & \\
\hline \multirow[t]{4}{*}{ MS4 } & F1 & GCATATCGTCTCATAGGAACA & 55 & 885 & 13 \\
\hline & R1 & GTTCATGGTTATTAATTCCAGAA & & & \\
\hline & $\mathrm{F} 2$ & CGAAGTGTACTACATGTCTCT & 55 & & \\
\hline & R2 & GGACTTTAATAAGTTACCTATAGT & & & \\
\hline \multirow[t]{4}{*}{ MS7 } & F1 & GTTGATCGTCCAGATGGAATT & 55 & 471 & 13 \\
\hline & R1 & GACTATCAGTATTACTGATTATAT & & & \\
\hline & F2 & CAATAGTAAAGGAAGATGGTCA & 55 & & \\
\hline & R2 & CGTCGCTITGTTTCATAATCTT & & & \\
\hline
\end{tabular}


Table 2 Factors associated with prevalence of Enterocytozoon bieneusi in farmed foxes in northern China

\begin{tabular}{|c|c|c|c|c|c|c|}
\hline Factor & Category & No. tested & No. positive & $\%(95 \% \mathrm{Cl})$ & OR $(95 \% \mathrm{Cl})$ & $P$-value \\
\hline \multirow[t]{3}{*}{ Region } & Jilin Province & 91 & 6 & $6.10(0.92-11.28)$ & Reference & 0.02 \\
\hline & Heilongjiang Province & 70 & 6 & $8.57(2.01-15.13)$ & $1.33(0.41-4.31)$ & \\
\hline & Hebei Province & 141 & 25 & $17.73(11.43-24.04)$ & 3.05 (1.20-7.77) & \\
\hline \multirow[t]{2}{*}{ Farming mode } & Indoor & 156 & 9 & $5.77(2.11-9.43)$ & Reference & $<0.0001$ \\
\hline & Outdoor & 146 & 38 & $26.03(18.91-33.15)$ & $5.75(2.67-12.39)$ & \\
\hline \multirow[t]{2}{*}{ Gender } & Male & 139 & 16 & 11.51 (6.21-16.82) & Reference & 0.72 \\
\hline & Female & 163 & 21 & 12.88 (7.74-18.03) & $1.14(0.57-2.28)$ & \\
\hline \multirow[t]{3}{*}{ Age } & Pre-weaned & 64 & 6 & $9.38(2.23-16.52)$ & Reference & 0.39 \\
\hline & Young & 180 & 21 & $11.67(6.98-16.36)$ & $1.28(0.49-3.32)$ & \\
\hline & Adult & 58 & 10 & 17.24 (7.52-26.96) & $2.01(0.68-5.94)$ & \\
\hline Total & & 302 & 37 & $12.25(8.55-15.95)$ & & \\
\hline
\end{tabular}

$200 \mu \mathrm{M}$ each deoxy-ribonucleoside triphosphate (dNTP), $0.4 \mu \mathrm{M}$ of each primer, $0.625 \mathrm{U}$ of Ex Taq DNA polymerase (TAKARA, Japan), and $2 \mu$ of DNA template. Cycling conditions consisted of preheating at $94{ }^{\circ} \mathrm{C}$ for $5 \mathrm{~min}$, then 35 cycles of $94{ }^{\circ} \mathrm{C}$ for $45 \mathrm{~s}$, annealing at $55-58{ }^{\circ} \mathrm{C}$ (Table 1 ) for $45 \mathrm{~s}$, and $72{ }^{\circ} \mathrm{C}$ for $1 \mathrm{~min}$, followed by a final extension at $72{ }^{\circ} \mathrm{C}$ for $10 \mathrm{~min}$. Positive and negative controls were included in each test. PCR products were visualized under UV light after electrophoresis in $2 \%$ agarose gels containing GoldView ${ }^{\text {TM }}$ (Solarbio, China).

\section{Sequencing and phylogenetic analyses}

Secondary PCR products were sequenced by Genscript Company (Nanjing, China). Sequences were aligned with reference sequences of $E$. bieneusi available in GenBank using the computer program Clustal X 1.83 and BLAST (http://www.ncbi.nlm.nih.gov/BLAST/). MEGA v5 (http:// www.megasoftware.net/) was used to infer phylogenetic trees by the neighbor-joining (NJ) method (Kimura 2parameter model). Bootstrapping (1000 replicates) was performed [28]. The E. bieneusi ITS genotypes were named according to the established nomenclature system [12].

\section{Statistical analysis}

The variation in E. bieneusi prevalence $(y)$ of foxes of different geographical location $(x 1)$, gender $(x 2)$, age $(x 3)$ and farming mode $(x 4)$ was analyzed using the 2 test in SAS version 9.1 (SAS Institute Inc., USA). In a multivariable regression analysis, each of these variables was included in a binary Logit model as an independent variable. The best model was judged by Fisher's scoring algorithm. All tests were two-sided, and results were considered statistically significant at $P<0.05$. Odds ratios (ORs) and their $95 \%$ confidence intervals (95\% CIs) were estimated to explore the strength of the association between E. bieneusi-positivity and each variable.

\section{Nucleotide sequence accession numbers}

Representative nucleotide sequences have been deposited into GenBank with the following accession

Table 3 Enterocytozoon bieneusi genotypes identified in farmed foxes from different farms in northern China

\begin{tabular}{|c|c|c|c|c|}
\hline Region & Farm ID & Age category (n) & $\begin{array}{l}\text { No. positive/no. } \\
\text { tested }(\%)\end{array}$ & Genotype (n) \\
\hline \multirow[t]{3}{*}{ Jilin Province } & 1 & Adults (2), Young (11), Pre-weaned (7) & $2 / 20(10 \%)$ & $\operatorname{NCF} 2(n=2)$ \\
\hline & 2 & Adults (8), Young (19), Pre-weaned (9) & $3 / 36(8.33 \%)$ & Peru8 $(n=1)$, Type IV $(n=1), \mathrm{D}(n=1)$ \\
\hline & 3 & Adults (1), Young (12), Pre-weaned (22) & $2 / 35(5.71 \%)$ & Type IV $(n=1), \mathrm{D}(n=1)$ \\
\hline \multirow[t]{2}{*}{ Heilongjiang Province } & 4 & Adults (7), Young (17), Pre-weaned (2) & 4/26 (15.38 \%) & $\begin{array}{l}\text { Peru8 }(n=1), \text { Type IV }(n=1), \text { CHN-DC1 } \\
(n=1), \operatorname{NCF} 2(n=1)\end{array}$ \\
\hline & 5 & Adults (9), Young (27), Pre-weaned (7) & $1 / 44(2.27 \%)$ & CHN-DC1 $(n=1)$ \\
\hline \multirow[t]{3}{*}{ Hebei Province } & 6 & Adults (9), Young (34), Pre-weaned (2) & $9 / 45(20.00 \%)$ & $\begin{array}{l}\text { Peru8 }(n=2), \operatorname{NCF} 5(n=2), \text { Type IV }(n=2) \\
\text { NCF1 }(n=2), \operatorname{NCF} 2(n=1)\end{array}$ \\
\hline & 7 & Adults (15), Young (33), Pre-weaned (9) & $5 / 57(8.77 \%)$ & $\operatorname{NCF} 2(n=4), \operatorname{NCF} 3(n=1)$ \\
\hline & 8 & Adults (7), Young (26), Pre-weaned (6) & $11 / 39(28.21 \%)$ & $\begin{array}{l}\mathrm{D}(n=2), \operatorname{NCF} 1(n=1), \operatorname{NCF} 2(n=5), \operatorname{NCF} 4 \\
(n=1), \operatorname{NCF} 6(n=1), \operatorname{NCF}(n=1)\end{array}$ \\
\hline Total & & Adults (68), Young (178), Pre-weaned (56) & $37 / 302(12.25 \%)$ & $\begin{array}{l}\text { Peru8 }(n=4), \text { Type IV }(n=5), \mathrm{D}(n=4), \mathrm{CHN}-\mathrm{DC} 1 \\
(n=2), \mathrm{NCF} 1(n=3), \mathrm{NCF} 2(n=13), \mathrm{NCF} 3(n=1), \mathrm{NCF} 4 \\
(n=1), \mathrm{NCF} 5(n=2), \operatorname{NCF} 6(n=1), \operatorname{NCF}(n=1)\end{array}$ \\
\hline
\end{tabular}


numbers: KT750157 to KT750167 for the ITS region, and KT844918 to KT844931 for the microsatellite loci (MS1, MS3, MS7).

\section{Results}

Prevalence of $E$. bieneusi in foxes

Of 302 fecal samples, 37 (12.25\%) were found to be $E$. bieneusi-positive by nested PCR amplification of the ITS region (Table 2). Enterocytozoon bieneusi was detected in each of the eight farms surveyed, and the highest infection rate was found in farm $8(28.21 \%, 11 / 39)$ (Table 3). There was no statistically significant difference in prevalence between males and females $(P=0.72)$ (Table 2). Adult foxes had a higher E. bieneusi prevalence compared with young foxes and pre-weaning foxes, but the difference was not significant $(P=0.39)$ (Table 2$)$. Statistically significant differences were observed among provinces (Table 2). Foxes raised outdoors (26.03\% positive, $95 \%$ CI 18.91-33.15) showed a significantly higher E. bieneusi prevalence compared to those raised indoors (5.77 \% positive, $95 \%$ CI 2.11-9.43, $P<0.0001$ ) (Table 2).

\section{Association between E. bieneusi positivity and exposure}

The univariate analysis summarized in Table 2 shows a strong relationship between farming mode and E. bieneusipositivity. The impacts of multiple variables on $E$. bieneusi were evaluated by a forward stepwise logistic regression analysis using Fisher's scoring technique. In the final model, only one variable (farming mode) had an effect on prevalence, described by the equation $y=3.8786-1.1977 x 4$. Farming mode has a strong effect on the risk of E. bieneusi (Table 2).

\section{Genetic characterizations and genotype distribution of $E$. bieneusi in foxes}

Analysis of the ITS region of E. bieneusi revealed four known genotypes, namely Peru 8, Type IV, CHN-DC1 and $\mathrm{D}[15,24,29]$. In addition, seven novel genotypes (NCF1-NCF7) were detected (Table 3). A comparative analysis of the ITS sequences showed that the four known genotypes sequences of the identified $E$. bieneusi isolates were identical to that of Peru 8 (GenBank accession no. KJ668721), Type IV (accession no. KJ651436), CHN-DC1 (accession no. KJ710333) and D (accession no. JF776168) sequences available in GenBank, respectively.

Of the novel genotypes, NCF2 $(n=13,35.14 \%)$ was the most common, being found in 5 farms (farm 1 from Jilin province, farm 4 from Heilongjiang province, farms 6,7 and 8 from Hebei province). The remaining novel genotypes were only present in Hebei province (Table 3).
Phylogenetic relationships of E. bieneusi ITS genotypes Phylogenetic analysis indicated that all the genotypes identified in this study belonged to group 1 (Fig. 1). NCF5 $(n=2)$, NCF6 $(n=1), \operatorname{NCF7}(n=1)$, Peru8 $(n=4)$ and $\mathrm{D}(n=4)$ were sub-classified into group $1 \mathrm{a}$, with NCF5 and NCF6 forming a new clade; Type IV $(n=5)$ was in group 1c; NCF1 $(n=3), \operatorname{NCF} 2(n=13), \operatorname{NCF} 3(n=1)$, NCF4 $(n=1)$ and CHN-DC1 $(n=2)$ were grouped into group 1b, with NCF1NCF4 forming a new clade (Fig. 1).

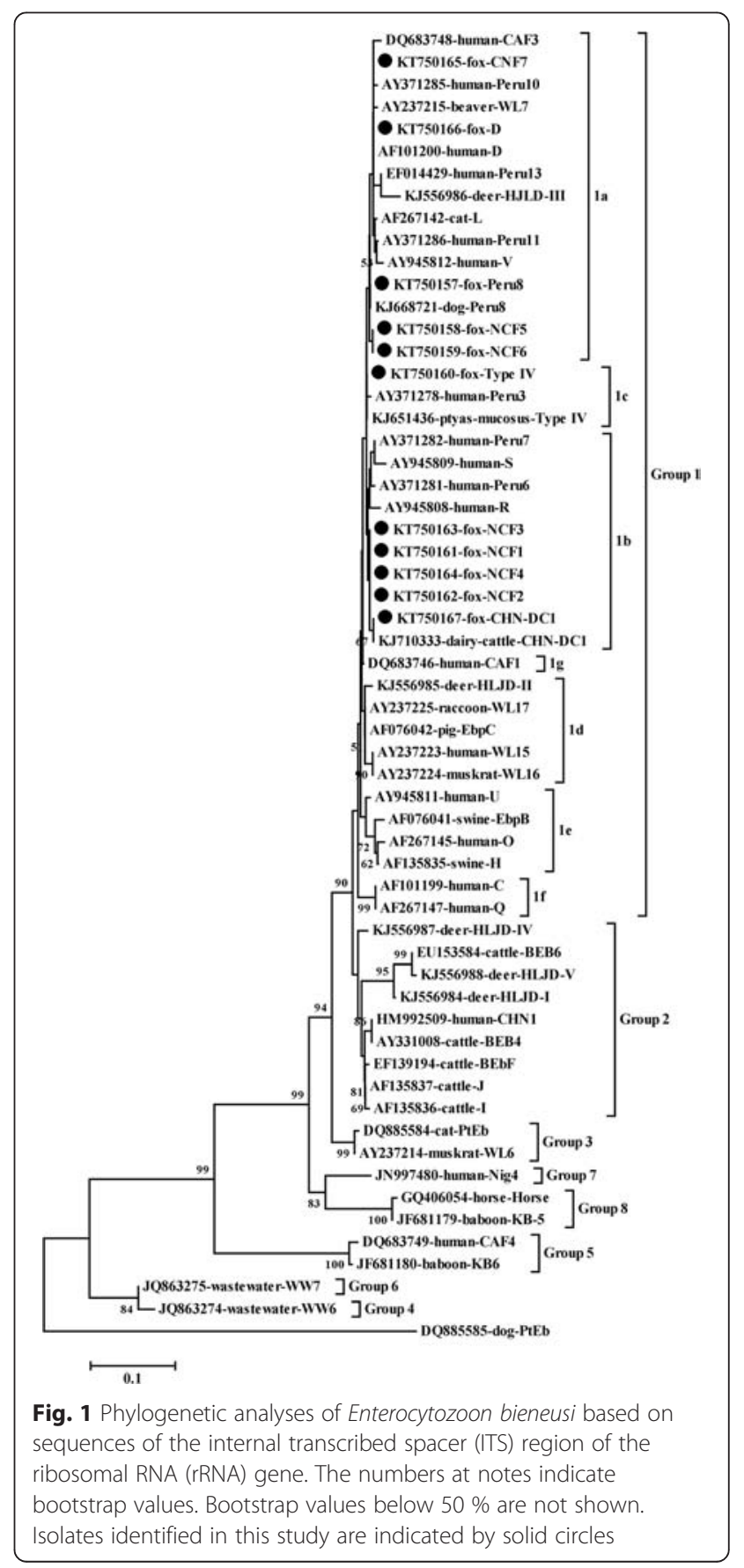




\section{Multilocus genotyping}

Three microsatellites (MS1, MS3 and MS7) loci and one minisatellite (MS4) locus were used to characterize the ITS-positive specimens. Twenty-one, 15, 0 and $17 E$. bieneusi isolates were successfully amplified at MS1, MS3, MS4, and MS7, respectively. Sequence analysis revealed 7, 2, 0, and 5 genotypes at loci MS1, MS3, MS4 and MS7, respectively. Ten E. bieneusi isolates were successfully amplified at all three microsatellites (MS1, MS3 and MS7) (Table 4).

\section{Discussion}

In the present study, 37 (12.25\%, $95 \%$ CI 8.55-15.95) out of 302 farmed foxes were E. bieneusi-positive by the nested PCR amplification of the ITS rDNA. This prevalence was lower than that in farmed foxes $(27.7 \%, 53 /$ 191) in Harbin City, China [27], and also lower than that in wild foxes in the Spain $(14.3 \%, 1 / 7)$ [29] and USA $(13.4 \%, 9 / 67)$ [30]. The different prevalence may be due to different geo-ecological conditions, sample collection time, animal husbandry practices, animal welfare, age distribution of the samples, as well as sample sizes.

Ingestion of contaminated water and food is the most important means of transmission of E. bieneusi [9]. Therefore, density of foxes in a farm and environmental sanitation may strongly affect on prevalence. Stocking density of foxes is generally higher in farms in Hebei and Heilongjiang provinces relative to those in Jilin province. This may explain why farms from Hebei and Heilongjiang had higher E. bieneusi prevalences than those from Jilin province. Farming mode is another risk factor associated with E. bieneusi infection in farmed foxes. Foxes raised outdoors had a significantly higher prevalence than those kept indoors, probably due to the fact that outdoor foxes have more opportunities to make contact with contaminated environments and animals than indoor foxes.
Four E. bieneusi ITS genotypes, namely D, EbpC, WL11, and WL15, have been detected in foxes in the world $[27,29,30]$. Only one of these, D, was among the four known genotypes that we found in farmed foxes. We also found genotypes Peru8, CHN-DC1 and Type IV, and an additional seven novel genotypes (NCF1-NCF7). Thus, eleven $E$. bieneusi genotypes were endemic in farmed foxes in northern China. All these genotypes belonged to group 1 analyzed by NJ methods (Fig. 1), which suggests that farmed foxes are potential sources of human microsporidiosis. NCF2 was the most frequent genotype found in the present study. In contrast, only genotype $\mathrm{D}$ was found in farmed foxes in Harbin City in a previous study [27] and in wild foxes in Spain [29], and genotypes WL13 and WL15 were the most common in wild foxes in the USA [30].

All of the four known genotypes, CHN-DC1, D, Type IV and Peru8, have been previously found in northern China [15, 24, 31]. For example, genotype D was found in golden takins (Budorcas taxicolor bedfordi) in Shannxi [13], HIV patients and non-human primates in Henan $[11,21]$, dairy cattle, sheep, goats, pig, cats and dogs in Heilongjiang [24, 31-33]. Type IV was present in HIV patients and non-human primates in Henan [21], cats and dogs in Heilongjiang [15]. Peru8 was found in nonhuman primates in Henan [11], dogs in Heilongjiang [15]. CHN-DC1 was identified only in dairy cattle in Heilongjiang [31]. These findings suggest crosstransmission of E. bieneusi between foxes, other animal species, and humans. More importantly, genotypes D and Peru8 have also been found in drinking water in China, which suggests that we should pay more attention to this mode of transmission of E. bieneusi.

Recently, multilocus sequence typing (MLST) has been used to further study the taxonomy and population genetics of E. bieneusi using MS1, MS3, MS4 and MS7 [13, 15, 34]. In the present study, amplification of at least one locus was successful for every isolate. Amplification of MS4 was unsuccessful in every case. All the three microsatellite loci

Table 4 Multilocus characterization of Enterocytozoon bieneusi isolates from farmed foxes in northern China

\begin{tabular}{|c|c|c|c|c|c|c|}
\hline \multirow[t]{2}{*}{ Code number } & \multirow[t]{2}{*}{ ITS genotype } & \multicolumn{5}{|c|}{ Multilocus genotypes } \\
\hline & & MS1 & MS3 & MS4 & MS7 & GenBank Accession Nos. \\
\hline 189 & NCF1 & FVIII & $\mathrm{FVI}$ & - & FXI & KT844921, KT844925, -, KT844931 \\
\hline 210 & NCF1 & FXI & FV & - & FX & KT844924, KT844926, -, KT844929 \\
\hline 247 & NCF2 & FX & $\mathrm{FVI}$ & - & FXI & KT844923, KT844925, -, KT844931 \\
\hline 251 & NCF2 & FVIII & $\mathrm{FVI}$ & - & $F X$ & KT844921, KT844925, -, KT844929 \\
\hline 272 & NCF2 & FXI & FV & - & FVIII & KT844924, KT844926, -, KT844930 \\
\hline 280 & NCF2 & $\mathrm{FXI}$ & $\mathrm{FV}$ & - & $F X$ & KT844924, KT844926, -, KT844929 \\
\hline 200 & Type IV & FXI & $\mathrm{FVl}$ & - & FX & KT844924, KT844925, -, KT844929 \\
\hline 89 & $\mathrm{D}$ & FVII & $\mathrm{FVI}$ & - & FX & KT844918, KT844925, -, KT844929 \\
\hline 276 & D & FIX & $\mathrm{FV}$ & - & FX & KT844922, KT844926, -, KT844929 \\
\hline 199 & Peru8 & FXII & $\mathrm{FVI}$ & - & FVIII & KT844919, KT844925, -, KT844930 \\
\hline
\end{tabular}


(MS1, MS3 and MS7) were only successfully amplified from ten isolates, revealing the presence of much diversity. These findings demonstrate the genetic diversity of E. bieneusi in farmed foxes.

\section{Conclusions}

The present study revealed the existence (12.25\%, 37/302) of zoonotic E. bieneusi infection in farmed foxes in Jilin, Heilongjiang and Hebei provinces, northern China. Foxes raised outdoors displayed a significantly higher $E$. bieneusi prevalence than those raised indoors. This study also found ITS genotypes D, Peru8, CHN-DC1 and Type IV, and seven novel genotypes (NCF1-NCF7) in farmed foxes, and further diversity was revealed at three microsatellite loci combining with microsatellite locus for the first time. These results suggest that control strategies are required to limit $E$. bieneusi infection in farmed foxes, and to prevent transmission to humans and other animals.

\section{Competing interests}

The authors declare that they have no competing interests.

\section{Authors' contributions}

$X Q Z$ and $X X Z$ conceived and designed the study, and critically revised the manuscript. XXZ,WC, ZLL, JGM, and WBZ performed the experiments. XXZ and WC analyzed the data. XXZ drafted the manuscript. QXY and QZ helped in study design, study implementation and manuscript preparation. All authors read and approved the final manuscript.

\section{Acknowledgments}

Project support was provided by the Science Fund for Creative Research Groups of Gansu Province (Grant No. 1210RJIA006) and the Agricultural Science and Technology Innovation Program (ASTIP) (Grant No. CAAS-ASTIP2014-LVRI-03). We thank Prof David Blair at the College of Marine and Environmental Sciences, James Cook University, Australia for improving the English language of the manuscript.

\section{Author details 730046, PR China. ${ }^{2}$ College of Animal Science and Technology, Jilin Medicine, Yangzhou, Jiangsu Province 225009, PR China. \\ Received: 14 October 2015 Accepted: 30 January 2016

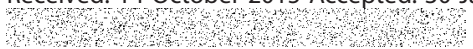

${ }^{1}$ State Key Laboratory of Veterinary Etiological Biology, Key Laboratory of Veterinary Parasitology of Gansu Province, Lanzhou Veterinary Research Institute, Chinese Academy of Agricultural Sciences, Lanzhou, Gansu Province Agricultural University, Changchun, Jilin Province 130118, PR China. ${ }^{3}$ Jiangsu Co-innovation Center for the Prevention and Control of Important Animal Infectious Diseases and Zoonoses, Yangzhou University College of Veterinary

\section{References}

1. Bakowski MA, Desjardins CA, Smelkinson MG, Dunbar TL, Lopez-Moyado IF, Rifkin SA, et al. Ubiquitin-mediated response to microsporidia and virus infection in C. elegans. PLoS Pathog. 2014;10:e1004200.

2. Li W, Li Y, Li W, Yang J, Song M, Diao R, et al. Genotypes of Enterocytozoon bieneusi in livestock in China: high prevalence and zoonotic potential. PLOS ONE. 2014;9:e97623.

3. Karim MR, Wang R, He X, Zhang L, Li J, Rume Fl, et al. Multilocus sequence typing of Enterocytozoon bieneusi in nonhuman primates in China. Vet Parasitol. 2014;200:13-23.

4. Zhao W, Zhang W, Wang R, Liu W, Liu A, Yang D, et al. Enterocytozoon bieneusi in sika deer (Cervus nippon) and red deer (Cervus elaphus): deer specificity and zoonotic potential of ITS genotypes. Parasitol Res. 2014;113:4243-50.
5. Santín M, Fayer R. Microsporidiosis: Enterocytozoon bieneusi in domesticated and wild animals. Res Vet Sci. 2011;90:363-71.

6. Desportes I, Le Charpentier Y, Galian A, Bernard F, Cochand-Priollet B, Lavergne A, et al. Occurrence of a new microsporidan: Enterocytozoon bieneusi n.g., n. sp., in the enterocytes of a human patient with AIDS. J Protozool. 1985;32:250-4.

7. Kondova I, Mansfield K, Buckholt MA, Stein B, Widmer G, Carville A, et al. Transmission and serial propagation of Enterocytozoon bieneusi from humans and Rhesus macaques in gnotobiotic piglets. Infect Immun. 1998;66:5515-9.

8. Deplazes P, Mathis A, Müller C, Weber R. Molecular epidemiology of Encephalitozoon cuniculi and first detection of Enterocytozoon bieneusi in faecal samples of pigs. J Eukaryot Microbiol. 1996;43:93S.

9. Bern C, Kawai V, Vargas D, Rabke-Verani J, Williamson J, Chavez-Valdez R, et al. The epidemiology of intestinal microsporidiosis in patients with HIV/AIDS in Lima, Peru. J Infect Dis. 2005;191:1658-64.

10. Tian GR, Zhao GH, Du SZ, Hu XF, Wang HB, Zhang LX, et al. First report of Enterocytozoon bieneusi from giant pandas (Ailuropoda melanoleuca) and red pandas (Ailurus fulgens) in China. Infect Genet Evol. 2015;34:32-5.

11. Karim MR, Wang R, Dong H, Zhang L, Li J, Zhang S, et al. Genetic polymorphism and zoonotic potential of Enterocytozoon bieneusi from nonhuman primates in China. Appl Environ Microbiol. 2014;80:1893-8.

12. Santín M, Fayer R. Enterocytozoon bieneusi genotype nomenclature based on the internal transcribed spacer sequence: a consensus. J Eukaryot Microbiol. 2009;56:34-8.

13. Zhao GH, Du SZ, Wang HB, Hu XF, Deng MJ, Yu SK, et al. First report of zoonotic Cryptosporidium spp., Giardia intestinalis and Enterocytozoon bieneusi in golden takins (Budorcas taxicolor bedfordi). Infect Genet Evol. 2015;34:394-401.

14. Hu Y, Feng Y, Huang C, Xiao L. Occurrence, source, and human infection potential of Cryptosporidium and Enterocytozoon bieneusiin drinking source water in Shanghai, China, during a pig carcass disposal incident. Environ Sci Technol. 2014:48:14219-27.

15. Karim MR, Dong H, Yu F, Jian F, Zhang L, Wang R, et al. Genetic diversity in Enterocytozoon bieneusi isolates from dogs and cats in China: host specificity and public health implications. J Clin Microbiol. 2014;52:3297-302.

16. Ma J, Li P, Zhao X, Xu H, Wu W, Wang Y, et al. Occurrence and molecular characterization of Cryptosporidium spp. and Enterocytozoon bieneusi in dairy cattle, beef cattle and water buffaloes in China. Vet Parasitol. 2015;207:220-7.

17. Wang L, Xiao L, Duan L, Ye J, Guo Y, Guo M, et al. Concurrent infections of Giardia duodenalis, Enterocytozoon bieneusi, and Clostridium difficile in children during a cryptosporidiosis outbreak in a pediatric hospital in China. PLoS Negl Trop Dis. 2013;7:e2437.

18. Du SZ, Zhao GH, Shao JF, Fang YQ, Tian GR, Zhang LX, et al, Cryptosporidium spp., Giardia intestinalis, and Enterocytozoon bieneusi in captive non-human primates in Qinling Mountains. Korean J Parasitol. 2015:53:395-402.

19. Thellier M, Breton J. Enterocytozoon bieneusiin human and animals, focus on laboratory identification and molecular epidemiology. Parasite. 2008;15:349-58.

20. Li N, Xiao L, Wang L, Zhao S, Zhao X, Duan L, et al. Molecular surveillance of Cryptosporidium spp., Giardia duodenalis, and Enterocytozoon bieneusiby genotyping and subtyping parasites in wastewater. PLoS Negl Trop Dis. 2012;6:e1809.

21. Wang L, Zhang H, Zhao X, Zhang L, Zhang G, Guo M, et al. Zoonotic Cryptosporidium species and Enterocytozoon bieneusi genotypes in HIVpositive patients on antiretroviral therapy. J Clin Microbiol. 2013;51:557-63.

22. Karim MR, Yu F, Li J, Li J, Zhang L, Wang R, et al. First molecular characterization of enteric protozoa and the human pathogenic microsporidian, Enterocytozoon bieneusi, in captive snakes in China. Parasitol Res. 2014;113:3041-8.

23. Li W, Diao R, Yang J, Xiao L, Lu Y, Li Y, et al. High diversity of humanpathogenic Enterocytozoon bieneusi genotypes in swine in northeast China. Parasitol Res. 2014;113:1147-53.

24. Li W, Li Y, Song M, Lu Y, Yang J, Tao W, et al. Prevalence and genetic characteristics of Cryptosporidium, Enterocytozoon bieneusi and Giardia duodenalis in cats and dogs in Heilongjiang province, China. Vet Parasitol. 2015;208:125-34.

25. Ye J, Xiao L, Wang Y, Guo Y, Roellig DM, Feng Y. Dominance of Giardia duodenalis assemblage $\mathrm{A}$ and Enterocytozoon bieneusi genotype BEB6 in sheep in Inner Mongolia, China. Vet Parasitol. 2015;210:235-9. 
26. Ye J, Xiao L, Li J, Huang W, Amer SE, Guo Y, et al. Occurrence of humanpathogenic Enterocytozoon bieneusi, Giardia duodenalis and Cryptosporidium genotypes in laboratory macaques in Guangxi, China. Parasitol Int. 2014;63:132-7.

27. Yang $Y$, Lin $Y, L i ~ Q$, Zhang $S$, Tao W, Wan Q, et al. Widespread presence of human-pathogenic Enterocytozoon bieneusi genotype $\mathrm{D}$ in farmed foxes (Vulpes vulpes) and raccoon dogs (Nyctereutes procyonoides) in China: first identification and zoonotic concern. Parasitol Res. 2015;114:4341-8.

28. Qin SY, Zhang XX, Zhao GH, Zhou DH, Yin MY, Zhao Q, et al. First report of Cryptosporidium spp. in white yaks in China. Parasit Vectors. 2014;7:230.

29. Galván-Díaz AL, Magnet A, Fenoy S, Henriques-Gil N, Haro M, Gordo FP, et al. Microsporidia detection and genotyping study of human pathogenic E. bieneusi in animals from Spain. PLoS ONE. 2014;9:e92289.

30. Sulaiman IM, Fayer R, Lal AA, Trout JM, Schaefer 3rd FW, Xiao L. Molecular characterization of microsporidia indicates that wild mammals harbor hostadapted Enterocytozoon spp. as well as human-pathogenic Enterocytozoon bieneusi. Appl Environ Microbiol. 2003;69:4495-501.

31. Zhao W, Zhang W, Yang F, Zhang L, Wang R, Cao J, et al. Enterocytozoon bieneusi in dairy cattle in the Northeast of China: genetic diversity of ITS gene and evaluation of zoonotic transmission potential. J Eukaryot Microbiol. 2015;62:553-60.

32. Zhao W, Zhang W, Yang D, Zhang L, Wang R, Liu A. Prevalence of Enterocytozoon bieneusi and genetic diversity of ITS genotypes in sheep and goats in China. Infect Genet Evol. 2015;32:265-70.

33. Zhao W, Zhang W, Yang F, Cao J, Liu H, Yang D, et al. High prevalence of Enterocytozoon bieneusi in asymptomatic pigs and assessment of zoonotic risk at the genotype level. Appl Environ Microbiol. 2014:80:3699-707.

34. Li W, Cama V, Akinbo FO, Ganguly S, Kiulia NM, Zhang X, et al. Multilocus sequence typing of Enterocytozoon bieneusi: lack of geographic segregation and existence of genetically isolated sub-populations. Infect Genet Evol. 2013;14:111-9.

\section{Submit your next manuscript to BioMed Central and we will help you at every step:}

- We accept pre-submission inquiries

- Our selector tool helps you to find the most relevant journal

- We provide round the clock customer support

- Convenient online submission

- Thorough peer review

- Inclusion in PubMed and all major indexing services

- Maximum visibility for your research

Submit your manuscript at www.biomedcentral.com/submit

C Biomed Central 